\title{
THE TRIANGULAR REPRESENTATION OF C. APOSTOL
}

\author{
RICHARD BOULDIN
}

\begin{abstract}
The existence of the triangular representation of C. Apostol is proved by arguments alternative to the original proof. The briefer development is, hopefully, more perspicuous than the original development.
\end{abstract}

1. Introduction. The purpose of this note is to give an alternative development of the triangular model of C. Apostol [1]. This model, which is a key theorem in much recent research [2], [3], [4], can be regarded as a continuation of the study of index theory for (bounded) operators on a complex Hilbert space. Points in the semi-Fredholm domain where the (orthogonal) projection onto the kernel of $T-\lambda$ fails to be continuous in the operator norm are called singular points. The remaining points in the semi-Fredholm domain are regular points. Each of the diagonal entries in the triangular representation of $T$ has simpler spectral properties on the above sets than $T$ has on the same sets. For example, one entry has the same singular points as $T$ but each singular point is an isolated eigenvalue.

By the use of some results from [8], which Apostol did not use, the triangular representation is obtained more quickly and more simply. A few of Apostol's constructions have been altered but the essential ideas are the same.

2. Preliminaries. Let $\mathcal{L}(H)$ denote all operators on the complex Hilbert space $H$. For $T \in \mathcal{L}(H)$ let $\operatorname{nul}(T-\lambda)$ be the dimension of the kernel of $T-\lambda$, denoted $\operatorname{ker}(T-\lambda)$, and let $\operatorname{def}(T-\lambda)$ be the codimension of the range of $T-\lambda$, denoted $(T-\lambda) H$. Provided $(T-\lambda) H$ is closed and either nul $(T-\lambda)$ or $\operatorname{def}(T-\lambda)$ is finite the operator $T-\lambda$ is semi-Fredholm; for such $\lambda$ the quantity nul $(T-\lambda)-\operatorname{def}(T-\lambda)$ is called the index of $T-\lambda$ and it is denoted ind $(T-\lambda)$. The set of all $\lambda$ for which $(T-\lambda)$ is semi-Fredholm is called the semi-Fredholm domain of $T$, denoted $\rho_{\mathrm{s}-\mathrm{F}}(T)$.

The form of the Index Theorem appropriate to this work is the following.

Index Theorem (Kato's version). For $T \in \mathcal{L}(H)$ and $\mu \in \rho_{\mathrm{s}-\mathrm{F}}(T)$ there is a neighborhood of $\mu$, say $G$, so that the following are true:

(i) $G \subset \rho_{\mathrm{s}-\mathrm{F}}(T)$ and $\lambda \in G$ implies $\operatorname{nul}(T-\lambda) \leqslant \operatorname{nul}(T-\mu) \operatorname{def}(T-\lambda)$ $\leqslant \operatorname{def}(T-\mu)$, ind $(T-\lambda)=\operatorname{ind}(T-\mu)$

(ii) $\operatorname{nul}(T-\lambda)$ and $\operatorname{def}(T-\lambda)$ are constant $G \backslash\{\mu\}$;

(iii) provided $\operatorname{nul}(T-\lambda)<\infty, \operatorname{nul}(T-\lambda)$ is constant on $G$ if and only if

Received by the editors March 3, 1975 and, in revised form, July 1, 1975 and September 8, 1975. AMS (MOS) subject classifications (1970). Primary 47A10; Secondary 47A65.

Key words and phrases. Semi-Fredholm, model, operator matrix.

( ) American Mathematical Society 1976 
$\operatorname{ker}(T-\mu) \subset \cap\left\{(T-\mu)^{k} H: k=1,2, \ldots\right\} ;$

(iv) provided $\operatorname{def}(T-\mu)<\infty, \operatorname{def}(T-\lambda)$ is constant on $G$ if and only if $\operatorname{ker}(T-\mu)^{*} \subset \cap\left\{\left(T^{*}-\bar{\lambda}\right)^{k} H: k=1,2, \ldots\right\}$.

Proof. Parts (i) and (ii) are well known; part (iii) is Problem 5.32 of $[8, \mathrm{p}$. 242 ] and (iv) results from applying (iii) to $(T-\lambda)^{*}$.

3. A result in index theory. The following result can be regarded as a refinement of (i), (ii) and (iii) of the Index Theorem. The (orthogonal) projection of $H$ onto $K$ is denoted $P_{K}$ and the orthogonal complement of $K$ in $H$ is $H \ominus K$. We denote $P_{K} T$ restricted to $K$ by $T_{K}$.

TheOREM. Take $T \in \mathcal{L}(H)$ and $\mu \in \rho_{\mathrm{s}-\mathrm{F}}(T)$. Then

$$
Q=\lim _{\lambda \rightarrow \mu} P_{\operatorname{ker}(T-\lambda)}
$$

exists in the operator norm, $Q$ is the orthogonal projection onto $\operatorname{ker}(T-\mu)$ $\cap \cap_{n}(T-\mu)^{n} H$, and

$$
\operatorname{dim}(\operatorname{ker}(T-\mu) \ominus Q H)<\infty .
$$

Proof. The proof is first given in the important case that $(T-\mu) H=H$. For $G$ chosen according to the Index Theorem and $\lambda \in G$ the operator $(T-\lambda)(T-\lambda)^{*}$ is onto. Since it is selfadjoint, it is invertible and $R(\lambda)$ $=(T-\lambda)^{*}\left((T-\lambda)(T-\lambda)^{*}\right)^{-1}$ is easily seen to be a right inverse for $(T-$ $\lambda)$. It follows that $I-R(\lambda)(T-\lambda)=P_{\operatorname{ker}(T-\lambda)}$ and consequently $\lim _{\lambda \rightarrow \mu} P_{\text {ker }(T-\lambda)}=P_{\text {ker }(T-\mu)}$.

Put $Y=\bigcap_{k}(T-\mu)^{k} H$. Because $(T-\mu)$ is semi-Fredholm the space $Y$ is closed in $H,(T-\mu) Y=Y$ and $\operatorname{dim} \operatorname{ker}(T-\mu) \ominus(Y \cap \operatorname{ker}(T-\mu))<\infty$. For the case nul $(T-\mu)<\infty$, this is proved on p. 241 of [8] but it is also true for $\operatorname{def}(T-\mu)<\infty$. In fact, by using that $\operatorname{dim} \operatorname{ker}(T-\mu) \ominus\left((T-\mu)^{k} H\right.$ $\cap \operatorname{ker}(T-\mu))$ is finite if $\operatorname{def}(T-\mu)<\infty$ (see [7, Lemmas 3.5 and 2.2]), one shows that

$$
\operatorname{dim}(T-\mu)^{j} H \cap \operatorname{ker}(T-\mu) \ominus(T-\mu)^{j+1} H \cap \operatorname{ker}(T-\mu)>0
$$

occurs only a finite number of times. The argument on p. 241 of [8] applies to show that $(T-\mu) Y=Y, \operatorname{dim} \operatorname{ker}(T-\mu) \Theta(Y \cap \operatorname{ker}(T-\mu))<\infty$.

For $\lambda \neq \mu$ clearly $(T-\mu) \operatorname{ker}(T-\lambda)=\operatorname{ker}(T-\lambda)$ and so $\operatorname{ker}(T-\lambda)$ $\subset Y$. It follows that

$$
P_{\text {ker }(T-\lambda)}=P_{\text {ker }\left(T_{Y}-\lambda\right)} P_{Y}
$$

and the first paragraph shows that this has the desired limit.

The significance of the following less technical result may be clearer.

Corollary. Take $T \in \mathcal{L}(H)$ and $\mu \in \rho_{\mathrm{s}-\mathrm{F}}(T)$. The following two conditions are equivalent:

(i) $P_{\mathrm{ker}(T-\lambda)}$ is continuous at $\lambda=\mu$;

(ii) either nul $(T-\lambda)$ or $\operatorname{def}(T-\lambda)$ is a finite constant in some neighborhood of $\mu$. 
Proof. If $\operatorname{nul}(T-\lambda)$ is a finite constant in some neighborhood of $\mu$ then the Index Theorem shows that $\operatorname{ker}(T-\mu) \subset(T-\mu)^{k} H$ for $k=1,2, \ldots$ It is elementary to see the equivalence of

$$
\operatorname{ker}(T-\mu) \subset \bigcap_{k}(T-\mu)^{k} H
$$

and

$$
\operatorname{ker}\left(T^{*}-\bar{\mu}\right) \subset \bigcap_{k}\left(T^{*}-\bar{\mu}\right)^{k} H .
$$

By the preceding theorem $P_{\operatorname{ker}(T-\lambda)}$ is continuous at $\lambda=\mu$. If def $(T-\lambda)$ is a finite constant in a neighborhood of $\mu$ then the argument is similar.

If $P_{\operatorname{ker}(T-\lambda)}$ is continuous at $\lambda=\mu$ then $(*)$ above holds. Hence $(* *)$ also holds and (ii) above follows from the Index Theorem.

4. A spectral theory result. Let $\rho_{\mathrm{s}-\mathrm{F}}^{\mathrm{r}}(T)\left(\rho_{\mathrm{s}-\mathrm{F}}^{\mathrm{s}}(T)\right)$ denote the regular (singular) points in the semi-Fredholm domain of $T$. Let $H_{r}(T)$ be the smallest subspace containing $\left\{\operatorname{ker}(T-\lambda): \lambda \in \rho_{\mathrm{s}-\mathrm{F}}^{\mathrm{r}}(T)\right\}$; let $H_{l}(T)$ be the smallest subspace containing $\left\{\operatorname{ker}(T-\lambda)^{*}: \lambda \in \rho_{\mathrm{s}-\mathrm{F}}^{\mathrm{r}}(T)\right\}$; let $H_{o}(T)$ be the orthogonal complement of $H_{r}(T)+H_{l}(T)$. Denote the compressions of $T$ to $H_{r}(T)$, $H_{l}(T)$ and $H_{o}(T)$ by $T_{r}, T_{l}$ and $T_{o}$, respectively. If $\lambda$ is an isolated eigenvalue of $T$ then there is a corresponding spectral subspace for $T$ and the dimension of that subspace is the algebraic multiplicity of $\lambda$, denoted sp.dim. $(\lambda ; T)$. Let $\sigma_{0}(T)$ denote the set of isolated eigenvalues with finite algebraic multiplicity. By $\sigma(T), \rho(T), \rho_{r}(T)$ and $\rho_{l}(T)$ we denote the spectrum of $T$, the resolvent set of $T$, the set of $\lambda$ such that $(T-\lambda) H=H$ and the set of $\lambda$ such that $\operatorname{ker}(T-\lambda)=\{0\}$, respectively.

Apostol's triangular representation and its basic properties are established in the following

THEOREM. (i) $H_{r}(T)$ is orthogonal to $H_{l}(T)$;

(ii) $H_{r}(T)$ and $H_{r}(T)+H_{o}(T)$ are invariant under $T$;

(iii) $\rho_{\mathrm{s}-\mathrm{F}}(T) \subset \rho_{r}\left(T_{r}\right) \cap \rho_{l}\left(T_{l}\right)$;

(iv) $\rho_{\mathrm{s}-\mathrm{F}}^{\mathrm{r}}(T) \subset \rho\left(T_{o}\right)$;

(v) $\rho_{\mathrm{s}-\mathrm{F}}^{\mathrm{s}}(T) \subset \sigma_{0}\left(T_{o}\right)$;

(iv) $\sigma_{0}(T) \subset \rho\left(T_{r}\right) \cap \rho\left(T_{l}\right) \cap \sigma_{0}\left(T_{o}\right)$;

(vii) sp. dim. $\left(\lambda ; T_{o}\right)=$ sp. $\operatorname{dim} .(\lambda ; T)$ for $\lambda \in \sigma_{0}(T)$.

Proof. By (iii) and (iv) of the Index Theorem, either

$$
\operatorname{ker}(T-\lambda) \subset(T-\lambda) H \quad \text { or } \quad \operatorname{ker}(T-\lambda)^{*} \subset(T-\lambda)^{*} H
$$

for every $\lambda \in \rho_{\mathrm{S}-\mathrm{F}}^{\mathrm{r}}(T)$. If $\mu \neq \lambda$ then $(T-\mu) \operatorname{ker}(T-\lambda)=\operatorname{ker}(T-\lambda)$ and so $\left(\operatorname{ker}(T-\mu)^{*}\right)^{\perp}=(T-\mu) H \supset \operatorname{ker}(T-\lambda)$. Clearly (i) follows.

Conclusion (ii) is straightforward.

In order to prove (iii) we take $\mu \in \rho_{\mathrm{s}-\mathrm{F}}^{\mathrm{r}}(T)$. Let $Y$ be the smallest subspace containing $\left\{\operatorname{ker}(T-\lambda): \lambda \in \rho_{\mathrm{s}-\mathrm{F}}^{\mathrm{r}}(T) \backslash\{\mu\}\right\}$. Since $P_{\operatorname{ker}(T-\lambda)}$ is continuous at $\mu, Y=H_{r}(T)$; since $(T-\mu) \operatorname{ker}(T-\lambda)=\operatorname{ker}(T-\lambda)$ for $\mu \neq \lambda,(T-\mu) Y$ $=Y$. Thus $\mu \in \rho_{r}\left(T_{r}\right)$.

Now take $\mu \in \rho_{\mathrm{s}-\mathrm{F}}^{\mathrm{s}}(T)$. Since $(T-\mu) Y \supset \operatorname{ker}(T-\lambda)$ for $\lambda \in \rho_{\mathrm{s}-\mathrm{F}}^{\mathrm{r}}(T)$, 
it suffices to show $(T-\mu) Y$ is closed. This is immediate from [8, Theorem 1.1] provided $\operatorname{nul}(T-\mu)<\infty$. Assume that $\operatorname{def}(T-\mu)<\infty$. As shown in the second paragraph of the proof of the theorem in $\$ 3$, one knows that $\operatorname{dim} \operatorname{ker}(T-\mu) \ominus(Y \cap \operatorname{ker}(T-\mu))<\infty$. Thus, $\operatorname{dim}(\operatorname{ker}(T-\mu)+Y)$ $\ominus Y<\infty$ (see [7, Lemma 2.2], for example) and $\operatorname{ker}(T-\mu)+Y$ is closed. Thus, Lemma IV, 2.9 of [6] implies that $(T-\mu) Y$ is closed. Since the corollary in $\$ 3$ implies that $P_{\operatorname{ker}(T-\lambda)^{*}}$ is continuous at $\mu \in \rho_{\mathrm{s}-\mathrm{F}}^{\mathrm{r}}(T)$, the preceding argument also shows that $\rho_{\mathrm{s}-\mathrm{F}}(T) \subset \rho_{l}\left(T_{l}\right)$.

In order to prove (iv) we take $\lambda \in \rho_{\mathrm{s}-\mathrm{F}}^{r}(T)$ and we assume $\left(T_{o}-\lambda\right) g=0$ or equivalently $(T-\lambda) g \in\left(H_{o}(T)\right)^{\mathrm{P}}$. By the $T$-invariance of $H_{r}(T)$ $+H_{o}(T)$, we get $(T-\lambda) g \in H_{r}(T)$. Since $(T-\lambda) H_{r}(T)=H_{r}(T)$ there is some $f \in H_{r}(T)$ such that $(T-\lambda) f=(T-\lambda) g$. Since $(f-g) \in \operatorname{ker}(T-\lambda)$ $\subset H_{r}(T)$, it must be that $g=0$. An analogous argument shows that $\operatorname{def}\left(T_{o}-\lambda\right)=0$ and so $T_{o}-\lambda$ is invertible.

From (ii) of the Index Theorem and (iv) of this theorem it follows that $\mu \in \rho_{\mathrm{s}-\mathrm{F}}^{\mathrm{s}}(T)$ is isolated from $\sigma\left(T_{o}\right)$. Clearly $\mu$ is an eigenvalue and (v) is proved if we show that the algebraic multiplicity of $\mu$ is finite. Since $(T-\mu)\left(H_{r}+H_{o}\right)=H_{r}+\left(T_{o}-\mu\right) H_{o}$ and since the subspace on the left is closed, $\left(T_{o}-\mu\right) H_{o}$ must be closed. We assume $\operatorname{nul}(T-\mu)<\infty$ and we omit the analogous case that $\operatorname{def}(T-\mu)<\infty$. For the sake of contradiction, let $\left\{e_{1}, e_{2} \cdots\right\}$ be an orthonormal set in $\operatorname{ker}\left(T_{o}-\mu\right)$. Since $(T-\mu) e_{k} \in H_{r}(T)$ there is some $f_{k} \in H_{r}(T)$ with $(T-\mu) f_{k}=(T-\mu) e_{k}$; thus $\left\{e_{1}-f_{1}, e_{2}\right.$ $\left.-f_{2}, \ldots\right\}$ is contained in $\operatorname{ker}(T-\mu)$ contradicting that $\operatorname{nul}(T-\mu)<\infty$. Denote the spectral subspace associated with the isolated eigenvalue $\mu$ by $H(\mu)$. By Lemma 5.29 of [8, p. 239], $\left(T_{o}-\mu\right) H(\mu)$ is closed and so Theorem 5.10 of [8, p. 233], implies $\operatorname{nul}\left(T_{o}-\mu\right)\left|H(\mu)=\operatorname{nul}^{\prime}\left(T_{o}-\mu\right)\right| H(\mu)$. On the other hand, if $\operatorname{dim} H(\mu)=\infty$ then Theorem 5.28 of [8, p. 239], shows that $\operatorname{nul}^{\prime}\left(T_{o}-\mu\right)=\infty$. Hence the algebraic multiplicity of $\mu$ is finite.

From (v) it is clear that $\sigma_{0}(T) \subset \sigma_{0}\left(T_{0}\right)$. For $\mu \in \sigma_{0}(T)$ and $\lambda \in \rho_{\mathrm{s}-\mathrm{F}}^{\mathrm{r}}(T)$ we get $\left(T_{r}-\mu\right) \operatorname{ker}(T-\lambda)=(\lambda-\mu) \operatorname{ker}(T-\lambda)$. Since $|\mu-\lambda|$ is bounded away from zero, $T_{r}-\mu$ is bounded below. This and (iii) imply $\mu \in \rho\left(T_{r}\right)$. An analogous argument shows $\mu \in \rho\left(T_{l}\right)$.

In order to prove (vii) take $\mu \in \sigma_{o}(T)$. It is easy to see that $\rho(T)$ $\subset \rho\left(T_{r}\right) \cap \rho\left(T_{l}\right)$. Because $\left(T_{r}-\mu\right)^{j}$ is bounded below on each $\operatorname{ker}(T-\lambda)$ for $\lambda \in \rho_{\mathrm{s}-\mathrm{F}}^{\mathrm{r}}(T)$ by the distance from $\mu$ to $\sigma\left(T_{r}\right) \backslash\{\mu\},\left(T_{r}-\mu\right)^{j}$ is invertible. Similarly $\left(T_{l}-\mu\right)^{j}$ is invertible. Since the spectral subspaces for $T$ and $T_{o}$ corresponding to $\mu$ are finite dimensional, for $j$ sufficiently large nul $(T-\mu)^{j}$ and $\operatorname{nul}\left(T_{o}-\mu\right)^{j}$ are equal to the dimensions of the corresponding spectral subspaces. Relative to $H_{r}(T) \oplus H_{o}(T) \oplus H_{l}(T)$ the matrix of $(T-\mu)^{J}$ is

$$
\left(\begin{array}{ccc}
\left(T_{r}-\mu\right)^{j} & A & B \\
0 & \left(T_{o}-\mu\right)^{j} & C \\
0 & 0 & \left(T_{l}-\mu\right)^{j}
\end{array}\right) .
$$

Clearly this matrix is zero on $\left(f_{1}, f_{2}, f_{3}\right)$ if and only if $f_{3}=0,\left(T_{o}-\mu\right)^{j} f_{2}$ $=0$ and $\left(T_{r}-\mu\right)^{j} f_{1}+A f_{2}=0$. Thus $f_{3}+f_{2}+f_{1}=\left(I-\left(T_{r}-\mu\right)^{-j} A\right) f_{2}$ and $\operatorname{nul}(T-\mu)^{j}=\operatorname{nul}\left(T_{o}-\mu\right)^{j}$.

The author thanks the referee for his helpful comments. 


\section{RICHARD BOULDIN}

\section{REFERENCES}

1. C. Apostol, The correction by compact perturbation of the singular behavior of operators, Rev. Roumaine Math. Pures Appl. (to appear).

2. - Matrix models for operators (to appear).

3. C. Apostol and B. Morrel, On uniform approximation of operators by simple models (to appear).

4. C. Apostol and K. Clancey, Generalized inverses and spectral theory, Trans. Amer. Math. Soc. 215 (1976), 293-300.

5. P. A. Fillmore, J. G. Stampfli and J. P. Williams, On the essential numerical range, the essential spectrum, and a problem of Halmos, Acta Sci. Math. (Szeged) 33 (1972), 179-192. MR 48 \# 896.

6. S. Goldberg, Unbounded linear operators: Theory and applications, McGraw-Hill, New York, 1966. MR 34 \# 580.

7. M. A. Kaashoek, Ascent, descent, nullity and defect: A note on a paper of A. E. Taylor, Math. Ann. 172 (1967), 105-115. MR 36 \# 5719.

8. T. Kato, Perturbation theory for linear operators, Die Grundlehren der math. Wissenschaften, Band 132, Springer-Verlag, New York, 1966. MR 34 \#3324.

Department of Mathematics, University of Georgia, Athens, Georgia 30601 